\title{
Synthesis, structure and morphology of poly(dimethylsiloxane) networks filled with in situ generated silica particles
}

\author{
Ludivine Dewimille ${ }^{\mathrm{a}}$, Bruno Bresson ${ }^{\mathrm{b}}$, Liliane Bokobza ${ }^{\mathrm{a}, *}$ \\ ${ }^{a}$ Laboratoire de Physico-Chimie Structurale et macromoléculaire, UMR 7615, ESPCI, 10 rue Vauquelin, 75231 Paris Cedex 05, France \\ ${ }^{\mathrm{b}}$ Laboratoire de Physique Quantique, E.S.P.C.I., 10 rue Vauquelin, 75231 Paris Cedex 5, France \\ Available online 10 March 2005
}

\section{Introduction}

In recent years, elastomeric composites have been the subject of a huge scientific interest. The hybrid materials usually display improved bulk properties relative to the base polymer. In particular, the incorporation of filler particles leads to an increase in stiffness resulting from the inclusion of rigid particles in the soft matrix and also from additional cross-linking sites at the particle-matrix interface $[1,2]$.

In addition to the volume fraction of the particles, filler parameters such as specific primary particle and aggregate size, spatial morphology or 'structure' and surface characteristics are well known to have a strong influence on the mechanical response of the resulting material. On the other hand, full realization of the filler capability can only be achieved if the filler is well dispersed in the rubbery matrix.

Functional groups on the particle surface determine the interactions between the polymer and the filler. The level of the matrix reinforcement markedly depends on the extent of interaction between the organic and inorganic phases. Although excessive polymer-filler bonding would be detrimental, some degree of interaction is desirable to assist filler dispersion and optimize the physical characteristics of the composites [3].

The reinforcing qualities of conventional fillers such as carbon blacks and silicas have been widely demonstrated in the literature. Filler particles are usually blended into the polymers before the cross-linking reaction. The particles tend to agglomerate and the resulting materials are rather inhomogeneous. The use of silane coupling agents, in combination with silica, in nonpolar polymers, is commonly used to help dispersion essentially in silica-filled hydrocarbon polymers where the interfacial adhesion between the two phases is poor.

Polysiloxanes polymers are an interesting class of materials and poly(dimethylsiloxane) (PDMS), with a repeat unit $\left[-\mathrm{Si}\left(\mathrm{CH}_{3}\right)_{2} \mathrm{O}-\right]$ is the most commonly used member of this class. However, PDMS elastomers exhibit very poor mechanical properties, with particularly low tensile strengths. So they need to be reinforced by mineral fillers in order to improve mechanical properties required in almost all commercial applications. PDMS is traditionally reinforced with silica and the chemical bonding between the two phases is ensured via hydrogen bonds between the silanols on the silica surface and the oxygen atoms of the polymer chains. The interactions between the silica filler and the PDMS network can be modified by a surface treatment of the mineral. Two approches of changing surface characteristics are frequently applied in rubber industry: surface modification by physical adsorption of some chemicals on the filler surface and permanent surface 
modification by passivating part of the silanol groups present on the surface of the particle.

In addition to the conventional technique of blending fumed silica into the elastomer prior to cross-linking, reinforcement of PDMS has also been obtained by in situ generated filler particles. This technique uses the sol-gel method based on the polymerization of inorganic precursors, the most common being the tetraethoxysilane (TEOS). Pioneered by Mark for essentially silica filling of silicone rubbers [4-9], the sol-gel process has been successfully extended to various other polymers [10-20]. The in situ organic-inorganic systems are often called nanocomposites on account of the small size of the generated structures.

The work previously reported on the reinforcement of PDMS by in situ generated silica, is essentially related to silicone elastomers formed by the use of functionallyterminated PDMS fluids that undergo catalyzed crosslinking reactions. Typically, silica precipitation can be carried out before or after or alternatively during curing [21]. In the third procedure, hydroxyl-terminated PDMS chains are blended with enough TEOS which will simultaneously end-link the precursor chains and provide the silica particles.

Two reactions are generally used to describe the sol-gel synthesis of silica from TEOS:

$$
\begin{aligned}
& \mathrm{Si}\left(\mathrm{OC}_{2} \mathrm{H}_{5}\right)_{4}+4 \mathrm{H}_{2} \mathrm{O} \stackrel{\text { hydrolysis }}{\longrightarrow} \mathrm{Si}(\mathrm{OH})_{4}+4 \mathrm{C}_{2} \mathrm{H}_{5} \mathrm{OH} \\
& \mathrm{Si}(\mathrm{OH})_{4}+\mathrm{Si}(\mathrm{OH})_{4} \stackrel{\text { condensation }}{\longrightarrow}(\mathrm{HO})_{3} \mathrm{Si}-\mathrm{O}-\mathrm{Si}(\mathrm{OH})_{3}+\mathrm{H}_{2} \mathrm{O}
\end{aligned}
$$

This process, which can be carried out at relatively low temperatures in host polymeric matrices, leads to samples exhibiting excellent mechanical properties and optical transparency.

The generated inorganic structures highly depend on the hydrolysis and condensation conditions and essentially on the nature of the catalyst used to accelerate the gelation process. The role played by the catalyst in the sol-gel processing of silica has been thoroughly examined by Pope and Mackenzie [22]. On the other hand, the mechanisms and kinetics of the hydrolysis-condensation reactions have been widely discussed on the basis of the water content and on the $\mathrm{pH}$ dependence of the gelation process $[23,24]$.

Large spherical particles are expected in the case of basecatalyzed reaction while linear chain growth is suggested via acid catalysis. Silica polymerization in the solution precursor is generally described by models of nucleation, growth and aggregation. Kinetic models based on fractal geometry concepts were developed and applied for the analysis of small-angle X-ray profiles scattering profiles in order to gain information on the morphology of the inorganic entities.

A wide range of complex structures were obtained in in situ silica-filled poly(dimethylsiloxane) networks prepared by various synthetic protocols $[7,11]$. Nevertheless, the typical fractal patterns and morphologies described in the case of the polymerization of silica in solution are not exactly those observed when the polymerization is carried out in PDMS [7]. This is most probably due to the constraints provided by the polymer environment.

This paper only concentrates on the sol-gel process carried out under two different catalytic conditions in an already-formed network by using a synthetic protocole different from those previously reported. Generating silica structures grow within a preformed PDMS network would prevent the formation of large silica aggregates. We will deal with the synthesis of the filled systems, silica structure and morphology analyzed by SAXS, SANS and transmission electron microscopy and will focus on the polymerfiller interface which plays a crucial role on the mechanical properties of the resulting materials. These mechanical properties will be discussed in a subsequent paper.

\section{Experimental}

\subsection{Sample preparation}

All the materials are used as received except for the cross-linking agent (1,3,5,7-tetramethylcyclotetrasiloxan) supplied by Gelest which contains $2 \%$ of chlorosilane thus removed by distillation.

Vinyl-terminated PDMS $\left(M_{\mathrm{w}}=17,200\right)$ (from Gelest) previously vacuum-dried during two or three hours to remove moisture, is mixed for half an hour with a small amount of platinum-divinyltetramethyldisiloxane used as the catalyst. Purified cross-linking agent, in excess of $40 \%$ with regard to the stoechiometric balance, is then added to the mixture under magnetic stirring just for a few minutes to avoid cross-linking reaction during mixing. The reacting mixture is slowly cast into a Teflon mold and left overnight at room temperature then at $80^{\circ} \mathrm{C}$ for one day for complete curing. The films are extracted with toluene for three days to remove any unreacted materials. The sol fractions are between 5 and $6 \%$.

In the usual in situ precipitation protocol, sheets of PDMS networks are swollen in TEOS then immersed in water containing a given catalyst [11]. In a recent work [25], the unfilled polymer network is swollen in TEOS containing $1 \mathrm{wt} \%$ of a tin salt (dibutyltin diacetate or dibutyltin dilaurate), the water required for the hydrolysis process being absorbed from the air.

In our in situ approach, the PDMS films were swollen in TEOS in the presence, as in the work of Rajan et al. [25], of a tin catalyst: dibutyltin diacetate (DBTDA) or dibutyltin dilaurate (DBTDL) at $3 \mathrm{wt} \%$ instead of $1 \mathrm{wt} \%$. The swelling time determines the degree of TEOS absorption and thus the filler loading. Both the TEOS-swollen film and a beaker containing water were placed for $24 \mathrm{~h}$ into a dessiccator maintained at a constant temperature $\left(30^{\circ} \mathrm{C}\right)$ thus exposing 

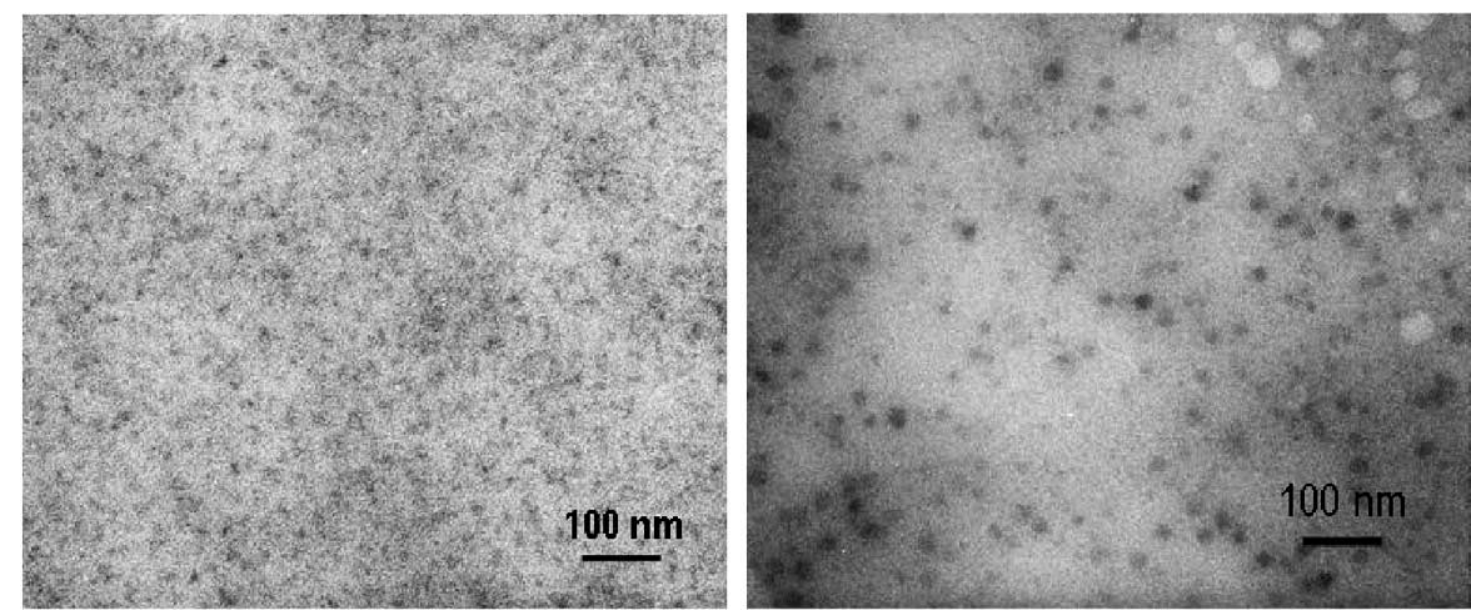

Fig. 1. TEM images of composites filled with $10 \mathrm{phr}$ of $\mathrm{SiO}_{2}$ precipitated into a PDMS network catalyzed by 3 wt $\%$ (DBTDL) (left) and by 3 wt $\%$ DEA (right).

the swollen film to saturated water vapor. The film was then vacuum-dried at $80^{\circ} \mathrm{C}$ for several days to constant weight in order to remove any alcohol generated from the reaction and also the remaining TEOS which has not been hydrolyzed.

For purpose of comparison, some samples were also prepared according to the procedure already reported $[7,11]$ which consists of immersing the TEOS-swollen network into an aqueous solution containing $3 \mathrm{wt} \%$ of DEA for one day. The films were then removed from solution and vacuum-dried as described above.

The amount of filler incorporated into the network was calculated from the weights of the films before and after the generation of the filler.

\subsection{Methods and measurements}

\subsubsection{Transmission electron microscopy (TEM)}

Ultrathin films of the samples were prepared using a diamond knife on an ultramicrotome at liquid nitrogen temperature. Specimens, around $60 \mathrm{~nm}$ thick were collected on standard copper grids, which were then coated with carbon and examined in transmission with a JEOL 100CXII electron microscope operating at $100 \mathrm{keV}$.

\subsection{Small-angle $X$-ray scattering (SAXS)}

Small angle X-ray scattering (SAXS) data were collected using the equipment provided by LURE at the University of Orsay. The wavelength of the beam was $1.489 \AA$ and the irradiated area was $1.5 \times 0.5 \mathrm{~mm}^{2}$. All data were collected at a sample-to-detector distance of $2.003 \mathrm{~m}$. The scattered intensity was obtained as a function of the scattering vector $q$, between $7.5 \times 10^{-3}$ and $0.3 \AA^{-1}$.

\subsection{Solid-state ${ }^{29}$ Si MAS NMR and ${ }^{1} H$ NMR}

The ${ }^{29}$ Si MAS NMR spectra were recorded on a Bruker CXP 300 spectrometer operating at $300 \mathrm{MHz}$. Magic angle spinning (MAS) was carried out at $7 \mathrm{kHz}$ spinning rate. Experiments were performed with a pulse of 3-4.5 $\mu \mathrm{s}$, and a repetition time of $5 \mathrm{~s}$ for cross-polarization experiments and $220 \mathrm{~s}$ without cross-polarization experiments, this latter delay being large enough to ensure almost complete relaxation of the silicon nuclei.

To study the magnetization relaxation, signals were recorded on a $300 \mathrm{MHz}$ Bruker spectrometer by standard Hahn-echo pulse sequence $((\pi / 2) \mathrm{x}, \tau,(\pi) \mathrm{y}$, acqu) with $\tau$ ranging from $6 \mu \mathrm{s}$ to $2 \mathrm{~ms}$. The magnetization decay corresponds to the envelope of the echoes. The $\pi / 2$ pulse length was $2 \mu \mathrm{s}$. All signals were recorded on resonance, and a cosine data set is obtained by proper adjustment of the receiver phase.

\subsection{Infrared spectra}

Infrared spectra were recorded with a Magna-IR 560 FTIR spectrometer equipped with a high-energy Ever-Glo source, a XT-KBr beamsplitter for spectral range coverage from 11,000 to $375 \mathrm{~cm}^{-1}$ and a DTGS detector. The spectra were recorded with a resolution of $4 \mathrm{~cm}^{-1}$ and an accumulation of 32 scans.

\subsection{Swelling measurements}

The samples were weighed, immersed in toluene and weighed again in order to determine its equilibrium swelling ratio $Q$, which is reached as the weight of the swollen sample does not increase anymore.

\section{6. $D S C$}

Pure PDMS and in situ silica filled PDMS were weighed accurately (5-15 mg) and were sealed in an aluminium pan. The TA DSC 2820 instrument was used to measure the melt transitions at a cooling and heating rate of $1.5^{\circ} \mathrm{C} / \mathrm{min}$ under 

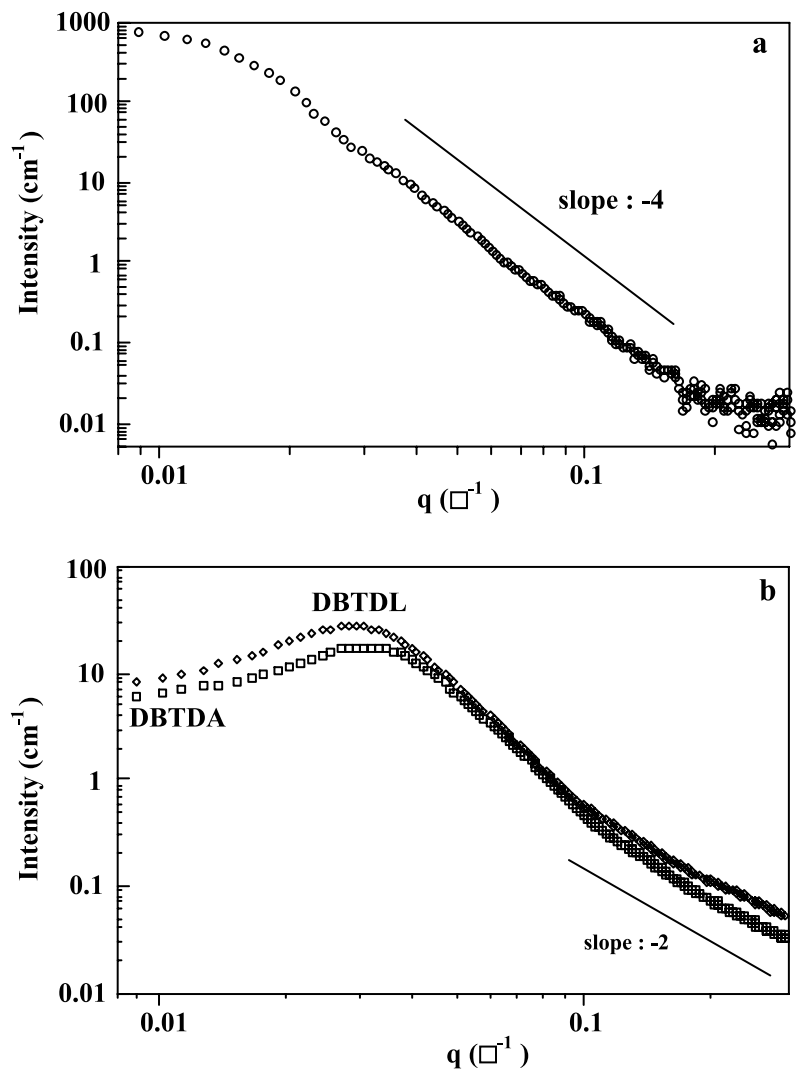

Fig. 2. Small-angle scattering profiles of composites filled with $20 \mathrm{phr}$ of silica particles generated under DEA catalyst (a); DBTDA and DBTDL catalysts (b).

$\mathrm{N}_{2}$ atmosphere. The crystallization temperature is measured at the maximum of the crystallization peak.

\section{Results and discussion}

\subsection{Characterization of the filler particles}

Fig. 1 shows TEM images in a DBTDL- and a DEAcatalyzed samples filled with $10 \mathrm{phr}$ of in situ silicas. While

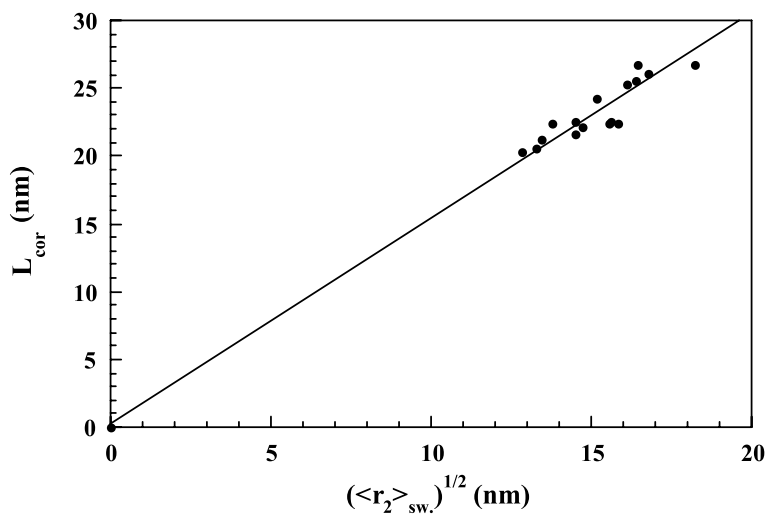

Fig. 3. Dependence of the characteristic length on the square root of meansquared end-to-end distance of a network chain in the swollen state. silica particles are uniformly dispersed in the polymer phase in both samples, quite different inorganic structures are revealed. The particles generated using $3 \mathrm{wt} \%$ DEA are seen to be spherical (diameter around $25 \mathrm{~nm}$ ) and larger than those generated using $3 \mathrm{wt} \%$ (DBTDL) as catalyst which in turn give rise to a highly porous material.

Small-angle X-ray scattering (SAXS) has been shown to yield important characteristics of the filler structures such as primary particle size, surface roughness as well as the size and the fractal dimension of larger scattering entities (aggregates and agglomerates) [7,8,26-28]. Additionally, some information regarding growth processes of the inorganic phase has been deduced from the analysis of scattering profiles. Essentially, it has been established that acid-catalyzed polymerization of the alkoxide is expected to proceed via a reaction-limited cluster aggregation and produces structures exhibiting a mass fractal dimension $\mathrm{D} \sim 2$ while a base catalysis is expected to give rise to domain formation via nucleation and growth [7].

As seen in Fig. 2, the two types of catalysts used in this study, yields distinctive small-angle scattering profiles for PDMS/SiO2 composites. The slope of -4 observed for the base-catalyzed composite (Fig. 7(a)) indicates that the system follows Porod's law with uniformly dense objects characterized by smooth surface fractal dimension ( $\mathrm{Ds}=2$ ). Moreover, experimental data were fitted by the factor form of spherical objects and the best fitting is obtained by the adjustment of the parameters $R$ particles radius and $d$ density of the silica. The size polydispersity was described using a Log-Normale distribution. The determined radius $R$ was around $15 \mathrm{~nm}$, which is consistent with MET observations.

With both tin catalysts, the analysis of the high-q region leads a slope close to -2 , suggesting the presence of a polymeric structure. A correlation peak, observed at $q \sim 0.03 \AA^{-1}$ associated with a characteristic length, Lcor, of about $20 \mathrm{~nm}$, can be seen as a spatial-periodicity. The maximum of the correlation peak is slightly shifted to smaller $q$ with the amount of filler. In addition, an inflection located at $q \sim 0.074 \AA^{-1}$ for filler loadings less than $20 \mathrm{phr}$ is shifted to $0.11 \AA^{-1}$ for highly loaded samples. This inflection, corresponding to a length around $5 \mathrm{~nm}$, may be associated with the diameter of the primary particles varying with the mesh size of the swollen network in which they are generated. In our synthetic protocol, silica formation takes place in a swollen network, the network swelling ratio in TEOS determining the amount of filler. So it is tempting to correlate the characteristic length, Lcor, (determined qmax) to the swelling ratio in TEOS, $Q$, through the following expression:

$\langle r 2\rangle_{\text {sw. }}=Q 2 / 3\langle r 2\rangle 0=Q 2 / 3 C n l 2$

where $C$ is the characteristic ratio of a chain of $n$ bonds of length $l$ and $\langle\mathrm{r} 2\rangle 0$ is the mean-squared end-to-end distance of a network chain in the unperturbed state. The nice 


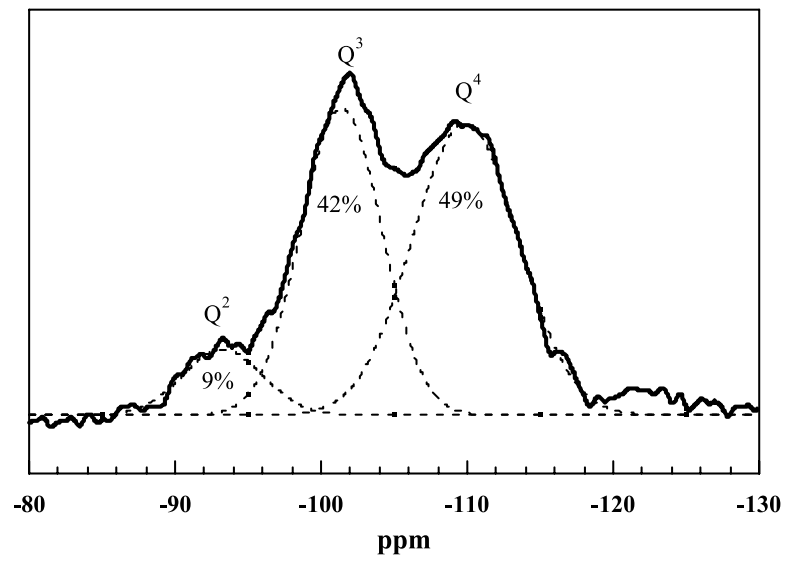

Fig. 4. Solid-state ${ }^{29}$ Si-NMR spectrum of a DBTDL- catalyzed PDMS composites containing $45 \mathrm{phr}$ of silica.

correlation obtained between Lcor and $\langle\mathrm{r} 2\rangle$ sw.1/2 (Fig. 3) leads to the belief the network itself imposes a length scale on the growth of the generated particles. This assumption is going to be checked with PDMS with varying molecular weight between cross-links.

As mentioned in the introduction, besides the particle size, the interfacial interaction between the organic and inorganic phases plays a crucial role in the reinforcement effects. In silica-filled PDMS samples, hydroxyl species, present in the silica structure, are involved in the interface; their amount and nature are expected to depend on the synthetic conditions.

Molecular spectroscopies are particularly well suited for a detailed analysis of the surface silanol hydroxyl groups (isolated and geminal, also denoted $Q_{3}$ and $Q_{2}, Q_{4}$ being related to $\mathrm{SiO}_{4}$ species). These surface sites are of particular interest since they determine the surface reactivity of silica with the environment.

Solid-state ${ }^{29} \mathrm{Si}$ MAS NMR spectroscopy allows an investigation of the chemical environment of the ${ }^{29} \mathrm{Si}$ atom. According to the literature [29], the resonances of the

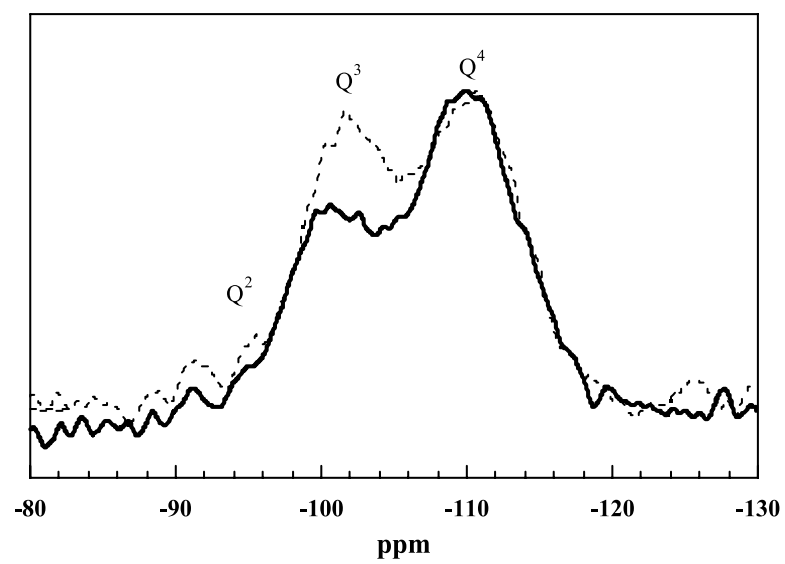

Fig. 5. Solid-state ${ }^{29} \mathrm{Si}-\mathrm{NMR}$ spectra of PDMS composites filled with $20 \mathrm{phr}$ of silica particles generated under DBTDA ( $\cdots)$ and DEA (-) catalysts.

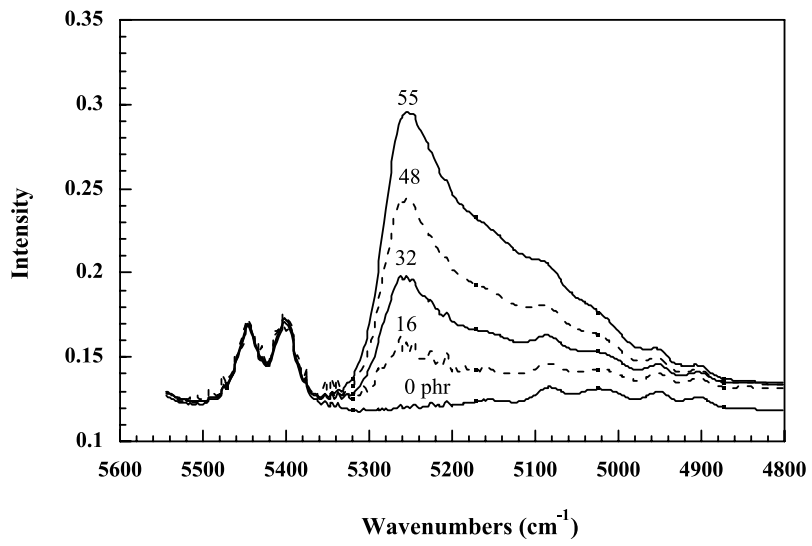

Fig. 6. Near-infrared spectra in the $4800-5600 \mathrm{~cm}^{-1}$ range of pure PDMS and DBTDA-catalyzed PDMS/SiO 2 composites: each curve is labelled with the amount of filler in phr (parts per hundred parts of rubber).

different species are located between 90 and 120 ppm (Fig. 4). The relative proportion of the different sites can be evaluated by spectra deconvolution.

The silica particles generated by the sol-gel process display a higher content of $Q_{3}$ species than that of $24 \%$ reported for fumed silica (A200 from Degussa) [30]. Moreover, at a same filler loading (20 phr), less $Q_{3}$ sites are found when the composite is prepared under base catalysis (Fig. 5). This would indicate that more condensed silica structures are obtained in the base-catalyzed samples.

Silanol groups, generally recognized as hydration sites, are expected to give rise, in infrared spectra, to strong absorption bands, mainly in the range $3800-3200 \mathrm{~cm}^{-1}$. Hydrogen bonding shifts the $\nu \mathrm{OH}$ wavenumber to lower values and broadens the absorption band. The infrared spectra of silicas obtained by different synthetic processes have been thoroughly discussed by Burneau et al. [29,31].

The infrared spectrum of a silica synthesized ex situ from TEOS in the presence of $3 \mathrm{wt} \%$ DBTDA with the water of hydrolysis simply absorbed from the atmosphere, displays a bround absorption around $3460 \mathrm{~cm}^{-1}$ due to water absorption with a shoulder around $3650 \mathrm{~cm}^{-1}$. This spectrum, which does not exhibit any isolated silanol (usually observed around $3747 \mathrm{~cm}^{-1}$ ), is similar to those obtained with Stber silicas [29,31].

States of physisorbed water can be characterized in the $5050-5350 \mathrm{~cm}^{-1}$ range by the combination of the bending and one of the stretching modes of the water molecule. Water physisorption can induce a change in the mechanical properties of silica-filled polymer composites, so the understanding of surface hydration of silica has received considerable attention. This water-silica interface greatly depends on the surface silanol distribution.

As seen in Fig. 6, the band located between 5000 and $5300 \mathrm{~cm}^{-1}$ in DBTDA-catalyzed $\mathrm{PDMS} / \mathrm{SiO}_{2}$ composites, increases with the amount of filler. The spectra exhibit an asymmetric absorption with a maximum at $5255 \mathrm{~cm}^{-1}$ and shoulders at lower wavenumbers around $5130 \mathrm{~cm}^{-1}$ as 


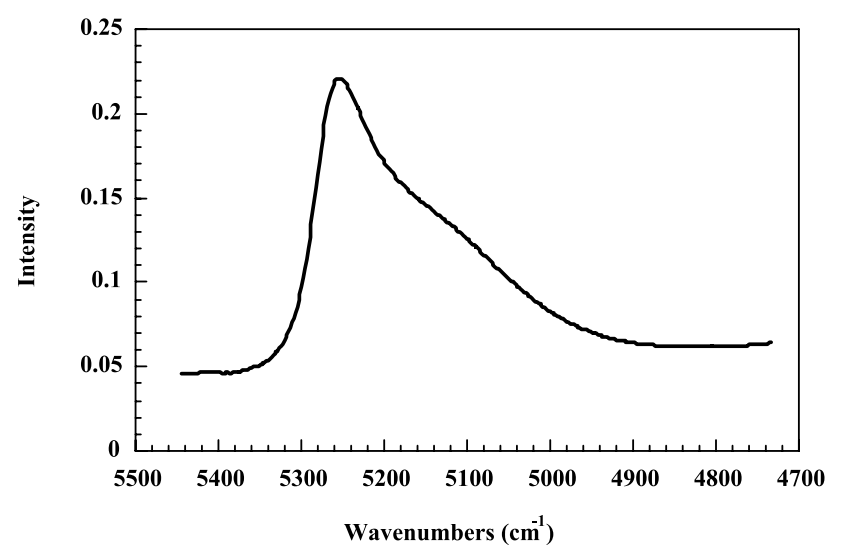

Fig. 7. Difference spectrum between the composite and the pure polymer spectra.

revealed by a spectral substraction of pure PDMS from the composite filled with $55 \mathrm{phr}$ of silica thus leading to the absorption profile of the $(\nu+\delta)$ band of water (Fig. 7). This typical profile has been associated with water adsorbed on inner silanols $[27,28]$. Contrary to internal or bulk groups, these inner silanols are accessible to a water molecule. The adsorbed water molecules can also be H-bonded to additional water molecules, thus explaining the occurrence of the band around $5130 \mathrm{~cm}^{-1}$.

Comparison of the profiles of the $(\nu+\delta)$ absorption band of water in various $\mathrm{PDMS} / \mathrm{SiO}_{2}$ composites containing a same filler loading (Fig. 8), shows that less water is adsorbed on a pyrogenic silica (A300 from Degussa). On the other hand, while a same spectral pattern is displayed by the two tin-catalyzed composites, a different water silicate interface is evidenced when the sol-gel process is conducted base catalysis. The intensity of the band around $5240 \mathrm{~cm}^{-1}$ decreases while a broad absorption grows around $5100 \mathrm{~cm}^{-1}$ indicating most likely clustering of water around the first adsorption sites.

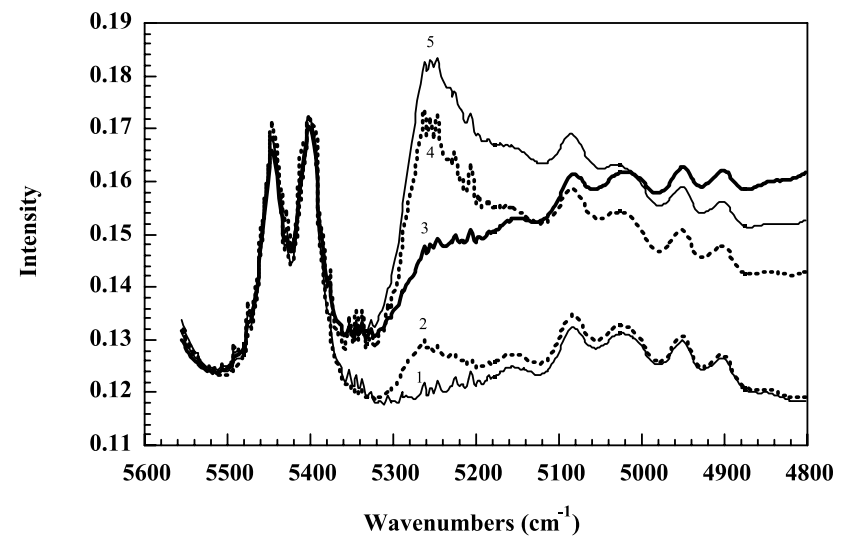

Fig. 8. Near-infrared spectra in the $4800-5600 \mathrm{~cm}^{-1}$ range of pure PDMS (1) and of PDMS composites filled with $20 \mathrm{phr}$ of silica: fumed silica of specific surface area of $300 \mathrm{~m}^{2} \mathrm{~g}^{-1}(2)$; in situ silica particles generated with the use of different catalysts: DEA (3); DBTDA (4); DBTDL (5).
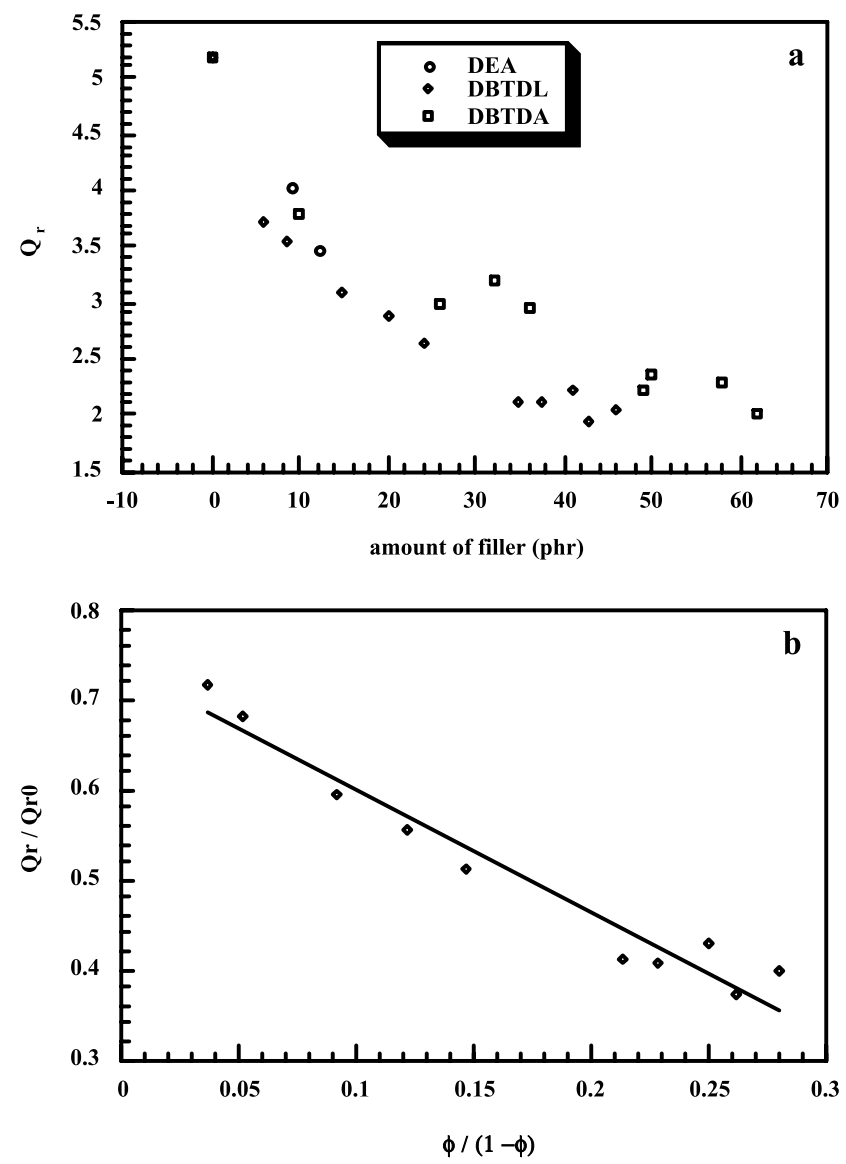

Fig. 9. Dependence of rubber phase swelling on the amount of filler (a) and plot of $Q_{\mathrm{r}} / Q_{\mathrm{r} 0}$ against filler loading expressed as volume ratio of filler to rubber (b).

\subsection{Characterization of the polymer-filler interface}

As it has been already demonstrated [4-9], silica particles in situ generated in PDMS networks, leads to substantial improvement in the moduli as well as in rupture properties. The increase in stiffness involves a hydrodynamic effect arising from the inclusion of rigid particles in the soft matrix and essentially to interfacial interactions between the organic and inorganic phases. These interactions, determined by the surface characteristics and the chemically active sites of the filler particles, largely govern the mechanical properties of the composite material. So, a characterization of the polymer-filler interface is essential for understanding the mechanical properties of the final materials.

\subsubsection{Swelling properties}

The degree of adhesion between polymer chains and filler particles is usually evaluated from equilibrium swelling in solvents. The extent of swelling at equilibrium is reduced in the case of an adsorption of polymer chains on the particle surface and may be enhanced with nonadhering fillers due to a dewetting of the particles and vacuole formation. 

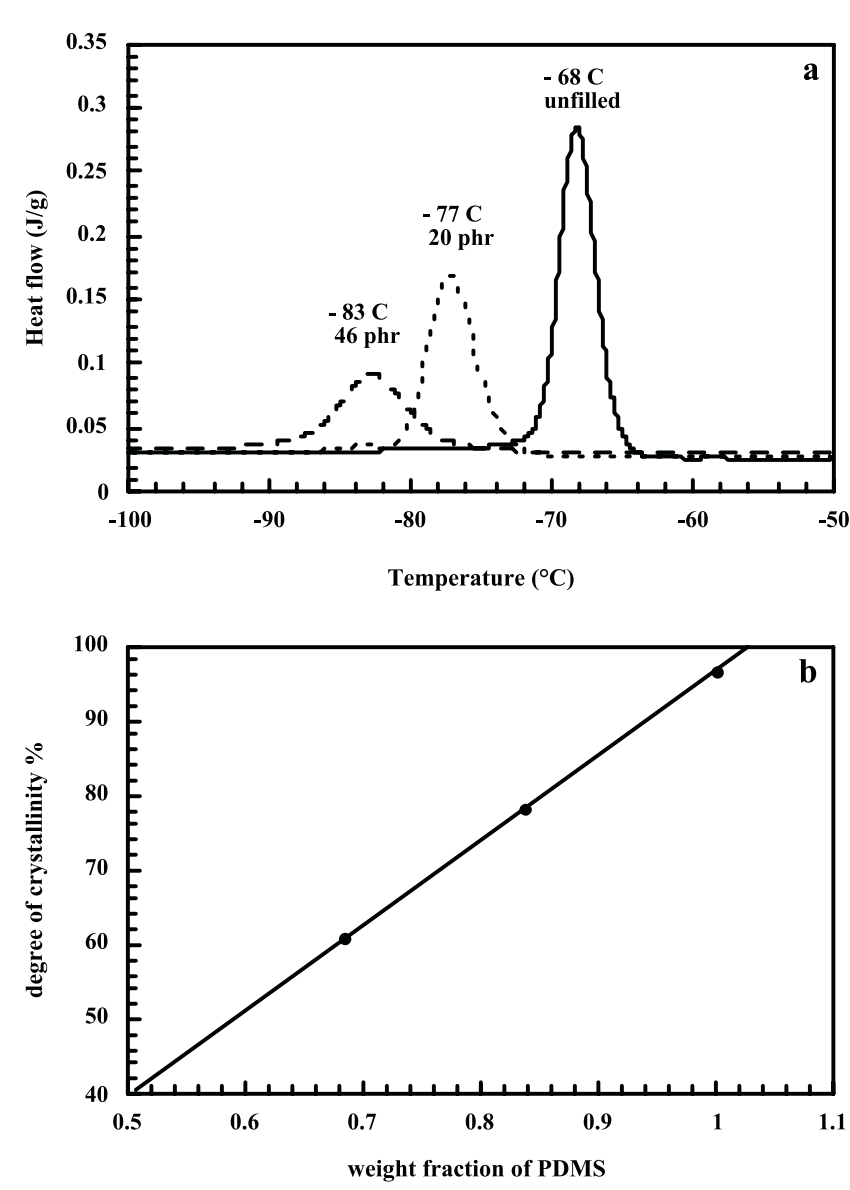

Fig. 10. Impact of the amount of filler (silica generated under DBTDL catalyst) on the crystallization peak (a) and on the degree of crystallinity (b).

As seen in Fig. 9(a), for each type of composite, the equilibrium swelling ratio in toluene of the rubber phase, $\mathrm{Q}_{\mathrm{r}}$, decreases with the amount filler, indicating a pronounced restriction in swelling in these in situ filled samples. $Q_{r}$ is defined by the following expression:

$Q_{\mathrm{r}}=(Q-\phi) /(1-\phi)$,

where $Q$ is the equilibrium swelling ratio of the composite, $\left(Q=V / V_{\mathrm{d}}, V\right.$ being the volume of the sample plus solvent and $V_{\mathrm{d}}$ that of the dry sample) and $\phi$ is the volume fraction of filler. Kraus [32,33] developed a theory for swelling of filled rubbers based on the assumption that the swelling is completely restricted at the particle surface and increases progressively at increases distances from the interface until reaching that of the unfilled rubber. It was shown that the swelling for a large number of filled vulcanizates obey an equation of the form:

$Q_{\mathrm{r}} / Q_{\mathrm{r} 0}=v_{\mathrm{r} 0} / v_{\mathrm{r}}=1-m \phi /(1-\phi)$

Where $Q_{\mathrm{r}}$ and $Q_{\mathrm{r} 0}$ are the rubber equilibrium swelling ratios for the filled and unfilled samples, respectively, $v_{\mathrm{r} 0}$ is the volume fraction of the unfilled rubber after swelling, $v_{\mathrm{r}}$ is the volume fraction of rubber in the gel of the filled

vulcanizate after swelling and:

$m=3 c\left(1-v_{\mathrm{r} 0}^{1 / 3}\right)+v_{\mathrm{r} 0}^{-1}$

$c$ is a constant characteristic of the filler, but independent of the polymer, the solvent or the degree of vulcanization. Such a representation is reported in Fig. 9(b) for the DBTDL-catalyzed composites.

\subsubsection{DSC measurements}

The large pronounced restriction in swelling discussed above can be considered as a result of adsorption interactions at the polymer-filler interface. This adsorption layer has also an impact on the thermal characteristics of the filled systems and essentially on the crystallization process.

Fig. 10(a) shows the crystallization peak for pure PDMS and for tin-catalyzed composites during cooling at $-1.5^{\circ} \mathrm{C} /$ min. The temperature of crystallization as well as the area under the peak (and consequently the degree of crystallinity) decreases with the filler content. This fact has already been reported by Aranguren in an investigation of unfilled PDMS and filled with pyrogenic silica in uncured and cured states [34].

The degree of crystallinity \%cryst. of the polymer is determined by:

$\%$ cryst $=\Delta H / \Delta H$ th

where $\Delta H$ is the enthalpy of crystallization measured for each sample and $\Delta H$ th, the enthalpy of fusion of PDMS taken as $37.43 \mathrm{~J} / \mathrm{g}$ [34]. Plotting the degree of crystallinity against the weight fraction of polymer in the sample (Fig. 10(b)), leads to a linear relationship whose intersect with the abscissa axis yields the polymer fraction $(15 \%)$ which is noncrystallizable, associated, by Aranguren [34], with bound rubber. This reduction in the ability of polymer to crystallize in the presence of filler may be regarded as the result of an increase in the apparent cross-linking density due to polymer-filler interactions. This interpretation is close to that reached by Patel et al. [35] in a study on the effect of the network cross-linking density on the crystallization characteristics of PDMS.

\subsubsection{NMR study}

Another way to characterize the effects of filler particles on the molecular motions of the polymer-chains at the polymer-filler interface is by ${ }^{1} \mathrm{H}$ NMR relaxation methods using spin-echo techniques. The transverse nuclear magnetic relaxation time, $T_{2}$, has been shown to be affected by physical and chemical cross-links in filled elastomers and subsequently to give access to the determination of the proportion of polymer with restricted mobility, in the adsorption layer surrounding the filler particles [36-39].

In a filled system where strong interactions are established between the active sites on the filler surface and polymer chains, $T_{2}$ values for the individual components can be extracted from the transverse magnetization 

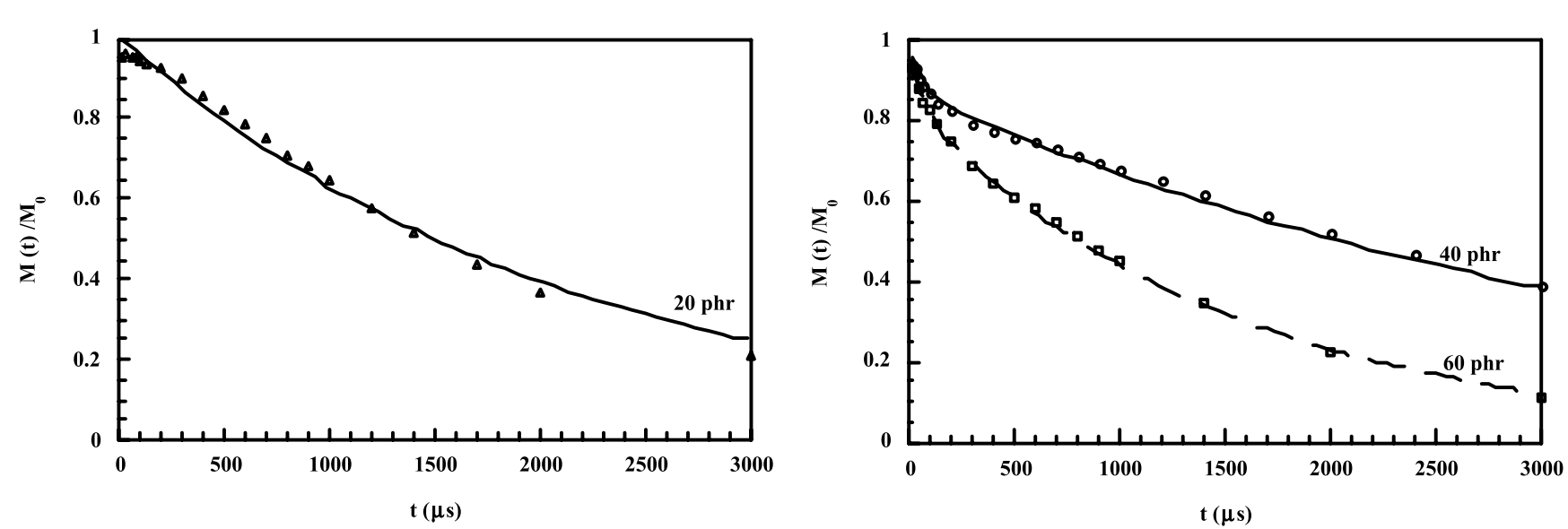

Fig. 11. Proton NMR relaxation data for tin-catalyzed $\mathrm{PDMS} / \mathrm{SiO}_{2}$ composites.

relaxation function $M(t)$, which can be written as:

$M(t)=M_{0} \exp \left[-t / T_{2}^{\mathrm{mob}}\right]+\left(1-M_{0}\right) \exp \left[-t / T_{2}^{\mathrm{rig}}\right]$

where $M_{0}$ is the fraction of mobile chains outside the adsorption layer whose dynamics are expected to be close to those of the unfilled rubber, $T_{2}^{\mathrm{mob}}$ and $T_{2}^{\mathrm{rig}}$ are the spin-spin relaxation times of the corresponding components.

The relaxation data obtained for samples prepared under tin catalysts are displayed in Fig. 11. For the composite filled with $20 \mathrm{phr}$ of silica, the data can be fitted satisfactorily by using a single exponential with a relaxation time of $1895 \mu \mathrm{s}$, higher than that of the unfilled network found equal to $2515 \mu \mathrm{s}$. The difference most probably arises from an increase in the cross-linking density ascribed to polymer-filler interactions. At higher filler loadings, the experimental data can be easily fitted with a sum of two exponential decay functions, corresponding to short $\left(T_{2}^{\mathrm{rig}}\right)$ and long $\left(T_{2}^{\mathrm{mob}}\right)$ relaxation times $\left(T_{2}^{\mathrm{rig}}=70 \mu \mathrm{s}\right.$, $T_{2}^{\mathrm{mob}}=1585 \mu \mathrm{s}$, for the sample filled with $60 \mathrm{phr}$, for example). The fraction of polymer of restricted mobility, given by $\left(1-M_{0}\right)$, is estimated to represent 15 and $16 \%$ for 40 and $60 \mathrm{phr}$ silica contents, respectively.

At a filler loading of $20 \mathrm{phr}$, the base-catalyzed composite, displays, contrary to the tin-catalyzed sample, two relaxation components: the $T_{2}$ value of the fast decaying component is around $20 \mu \mathrm{s}$ and corresponds to a fraction of 5\% of the total amount of PDMS. This behavior has to be connected to the different silica morphologies induced by the different catalysts. The condensed silica structures are obtained in the base-catalyzed sample should favor more polymer-filler contacts than a polymeric structure displayed under tin catalysts. In this latter case, adsorption interactions only take place in highly filled systems. As the radius, $R$, of the particles generated using $3 \mathrm{wt} \%$ DEA has been estimated around $10 \mathrm{~nm}$, the thickness, $e$, of the adsorption layer can be evaluated around $1.5 \mathrm{~nm}$ by the expression:

where $\omega$ is the fraction of immobilized polymer and $\phi$, the volume fraction of filler. This thickness is consistent with that already reported on previous studies [39].

\section{Conclusion}

PDMS / silica nanocomposites are synthesized by submitting already-formed networks to the sol-gel technique under the presence of two different catalysts. Various techniques are combined to investigate the morphology of the generated particles. In each case, the generated inorganic structures are uniformly dispersed in the polymer phase but different morphologies are revealed reflecting two different types of growth processes. The hydrophilic character of the filler surface is responsible of strong interactions with the polymer chains leading to a significant improvement in the mechanical properties of the composites which will be discussed in a second paper.

\section{References}

[1] Bokobza L. Macromol Symp 2001;169:243 and 171,163,2001.

[2] Bokobza L, Rapoport O. J Appl Polym Sci 2002;85:2301.

[3] Edwards DC. J Mater Sci 1990;25:4175.

[4] Wang S, Xu P, Mark JE. Rubber Chem Technol 1991;64:746.

[5] Mark JE. J Appl Polym Sci, Appl Polym Symp 1992;50:273.

[6] Wen J, Mark JE. Rubber Chem Technol 1994;67:806.

[7] McCarthy DW, Mark JE, Schaeffer DW. J Polym Sci, Part B: Polym Phys 1998;36:1167.

[8] McCarthy DW, Mark JE, Clarson SJ, Schaeffer DW. J Polym Sci, Part B: Polym Phys 1998;36:1191.

[9] Breiner JM, Mark JE, Beaucage G. J Polym Sci; Part B: Polym Phys 1999;37:1421.

[10] Tanahashi H, Osanai S, Shigekuni M, Murakami K, Ikeda Y, Kohjiya S. Rubber Chem Technol 1998;71:38.

[11] Yuan QW, Mark JE. Macromol Chem Phys 1999;200:206.

[12] Ikeda Y, Kohjiya S. Polym 1997;38:4417.

[13] Hashim AS, Azahari B, Ikeda Y, Kohjiya S. Rubber Chem Technol 1998;71:289.

[14] Kohjiya S, Ikeda Y. Rubber Chem Technol 2000;73:534.

[15] Kohjiya S, Murakami K, Iio S, Tanahashi T, Ikeda Y. Rubber Chem Technol 2001;74:16. 
[16] Murakami K, Iio S, Tanahashi T, Kohjiya S, Kajiwara K, Ikeda Y. KGK Kautsch Gummi Kunsttoffe 2001;54:668.

[17] Matejka L, Dukh O, Kolarik J. Polym 2000;41:1449.

[18] Matejka L, Dukh O. Macromol Symp 2001;171:181.

[19] Yoshikai K, Ohsaki T, Furukawa M. J Appl Polym Sci 2002;85:2053.

[20] Jang J, Park H. J Appl Polym Sci 2002;85:2074.

[21] Mark JE, Erman B. Rubber elasticity. A molecular primer. New York: Wiley-Interscience; 1988.

[22] Pope EJA, Mackenzie JD. J Non-Cryst Solids 1986;87:185.

[23] Pouxviel JC, Boilot JP, Beloeil JC, Lallemand JY. J Non-Cryst Solids 1987;89:345.

[24] Brinker CJ. J Non-Cryst Solids 1988;100:31.

[25] Rajan GS, Sur GS, Mark JE, Schaffer DW, Beaucage G. J Polym Sci, Part B: Polym Phys 2003;41:1897.

[26] Schaefer DW, Suryawanshi C, Pakdel P, Ilavsky J, Jemian PR. Physica A 2002;314:686.

[27] Schaefer DW, Chen C. Rubber Chem Technol 2002;75:773.

[28] Schaefer DW, Vu BTN, Mark JE. 2002;75:795.

[29] Legrand AP, Hommel H, Tuel A, Vidal A, Balard H, Papirer E, et al. Adv Colloid Interface Sci 1990;33:91.
[30] Lèonardelli S, Facchini L, Frètigny C, Tougne P, Legrand A. J Am Chem Soc 1992;114:6412.

[31] Burneau A, Gallas JP. Vibrational spectroscopies. In: Legrand AP, editor. The surface properties of silicas. Chichester, United Kingdom: Wiley; 1998. p. 147-234. 470 pages.

[32] Kraus G. Interactions between elastomers and reinforcing fillers. In: Kraus G, editor. Reinforcement of elastomers. New York: Wiley; 1965. p. $125-52$.

[33] Kraus G. Reinforcement of elastomers by carbon black, in Advances in polymer science 1971. p. 1557-237.

[34] Aranguren MI. Polymer 1998;39:4897.

[35] Patel M, Morrell PR, Skinner R. Macromol Symp 2002;180:109.

[36] Litvinov VM. Polym Sci USSR 1988;30:2250.

[37] Litvinov VM, Spiess HW. Makromol Chem 1991;192:3005.

[38] Litvinov VM, Spiess HW. Makromol Chem 1992;193:1181.

[39] Litvinov VM. Poly(dimethylsiloxane) chains at a silica surface. In: Auner N, Weis J, editors. Organosilicon chemistry II, from molecules to materials. Weinheim: VCH; 1996. p. 779-814. 